\title{
Battery Lifetime Prediction for Energy-Aware Computing
}

\author{
Daler Rakhmatov and Sarma Vrudhula \\ Center for Low Power Electronics \\ ECE Department, University of Arizona \\ Tucson, Arizona 85721 \\ daler,sarma@ece.arizona.edu
}

\author{
Deborah A. Wallach \\ Hewlett-Packard Western Research Laboratory \\ 250 University Avenue \\ Palo Alto, CA 94301 \\ deborah.wallach@hp.com
}

\begin{abstract}
Predicting the time of full discharge of a finite-capacity energy source, such as a battery, is important for the design of portable electronic systems and applications. In this paper we present a novel analytical model of a battery that not only can be used to predict battery lifetime, but also can serve as a cost function for optimization of the energy usage in battery-powered systems. The model is physically justified, and involves only two parameters, which are easily estimated. The paper includes the results of extensive experimental evaluation of the model with respect to numerical simulations of the electrochemical cell, as well as measurements taken on a real battery. The model was tested using constant, interrupted, periodic and non-periodic discharge profiles, which were derived from standard applications run on a pocket computer.
\end{abstract}

\section{Categories and Subject Descriptors}

C.4.5 [Performance of Systems]: Performance Attributes

\section{General Terms}

Performance, Experimentation

\section{Keywords}

Battery, modeling, low-power design

\section{INTRODUCTION}

Electrical energy in portable systems is commonly supplied by batteries of finite capacity. Once the battery is fully discharged the system can no longer remain on-line; therefore, it is important to have a model that can accurately predict battery behavior. A system designer can use such a model to construct task schedules and select task execution parameters, such as the operating voltage

\footnotetext{
*This work was partially carried out at the National Science Foundation's State/Industry/University Cooperative Research Centers' (NSF-S/IUCRC) Center for Low Power Electronics (CLPE). CLPE is supported by the NSF (Grant EEC-9523338), the State of Arizona, and a consortium of companies from the microelectronics industry (visit the CLPE web site http://clpe.ece.arizona.edu).
}

Permission to make digital or hard copies of all or part of this work for personal or classroom use is granted without fee provided that copies are not made or distributed for profit or commercial advantage and that copies bear this notice and the full citation on the first page. To copy otherwise, to republish, to post on servers or to redistribute to lists, requires prior specific permission and/or a fee.

ISLPED'02, August 12-14, 2002, Monterey, California, USA

Copyright 2002 ACM 1-58113-475-4/02/0008 ...\$5.00. and the clock frequency, so that the battery lasts as long as possible. A detailed discussion of battery-related issues in the context of portable electronic systems can be found in $[10,14]$.

In this paper, we present an analytical model that allows for lifetime predictions under time-varying load conditions. To demonstrate its quality, we compare our predictions with measured and simulated lifetime data using applications run on the Itsy pocket computer [15]. The simulated battery used in our study exhibits strong nonlinearity, and our model accurately accounts for these effects, including charge recovery. The predictions of our model for batteries which do not exhibit severe nonlinear effects are verified against a real battery.

Recently, there has been an increased interest in battery modeling on the part of both battery designers and battery users. Both simulation-based approaches and analytical efforts have been reported. To study batteries at the microscopic scale, one can perform a numerical simulation of partial differential equations that describe complex physical processes taking place inside an electrochemical cell $[4,7]$. Unfortunately, such simulators are slow, and consequently their utility within a CAD framework is limited. On the other hand, macroscopic models attempt to capture the battery behavior characteristics at a high level of abstraction. Researchers have proposed a simulation model based on a PSPICE equivalent circuit [8], a discrete-time VHDL specification [3], and a Markov chain [11]. Even though these simulation-based models can predict the battery lifetime for a given load profile, they do not provide a formal cost measure that can be used by system or application designers for lifetime optimization purposes. Also, such simulators may require the user to specify many model parameters, which are often difficult to obtain. On the other hand, analytical battery models provide a mathematical expression relating load conditions and a few battery parameters to the amount of delivered energy [5, 12]. Such models spell out key factors affecting battery performance, and consequently, can be employed as cost functions guiding a battery-aware system/application optimization process. However, these analytical models are inherently less accurate and less general than simulation models: they are obtained either empirically or by considering only limiting cases.

Our model offers accuracy and generality of a simulation-based approach yet has the advantages of an analytical model. A complex real battery is represented by a simpler equivalent battery, and a high-level analytical expression is derived on the basis of lowlevel analysis. One previous modeling attempt in this direction was reported in [13]. In this paper, we provide a new model which is substantially improved in terms of computational complexity, accuracy and usefulness.

\section{MODEL DESCRIPTION}

In general, the battery lifetime can be defined as the time when some quantity (measurable directly or indirectly) crosses a threshold value. Traditionally, such a quantity has been the voltage observed across the battery terminals, so that the lifetime is the time when the voltage drops below a cutoff level. Other quantities such as charge or energy can also be used. The model described here relates the battery lifetime to the time-varying load by accounting for the changes in concentration of the electroactive species inside 
the battery as a function of the load. This model is derived based on simplifications of complex phenomena, which differentiates our approach from empirical models.

We consider two main processes: electrochemical reaction at the electrode surface and ion diffusion in the electrolyte. We use the Faraday's law to describe the reaction behavior, and we assume that the diffusion process is one-dimensional in a finite-length region. The proposed battery model is an analytical solution of the system of two diffusion equations and three boundary conditions (see [2] for details):

$$
\begin{gathered}
-J(x, t)=D \frac{\partial C(x, t)}{\partial x}, \quad \frac{\partial C(x, t)}{\partial t}=D \frac{\partial^{2} C(x, t)}{\partial x^{2}}, \\
-J(0, t)=\frac{i(t)}{v F A}, \quad J(w, t)=0, \quad C(x, 0)=C^{*} \forall x .
\end{gathered}
$$

$J(x, t)$ and $C(x, t)$ are the flux and the concentration of electroactive species, respectively, at time $t$ and at distance $x$ from the electrode surface. $D$ is the diffusion coefficient, $v$ is the number of electrons involved in a chemical reaction, $A$ is the electrode area, $F$ is the Faraday's constant, $w$ is the length of the diffusion region, and $C^{*}$ is the initial concentration value assuming equilibrium. The battery is considered to be exhausted at time $t=L$, when $C(0, t)$ drops below some cutoff value $C_{\text {cutoff }}$.

Solving (1) involves direct and inverse Laplace transformations and manipulations of a theta function. Details of derivations are beyond the scope of this paper. The final solution is as follows:

$$
\begin{aligned}
& \alpha=\int_{0}^{L} i(\tau) d \tau+2 \sum_{m=1}^{\infty} \int_{0}^{L} i(\tau) e^{-\beta^{2} m^{2}(L-\tau)} d \tau, \\
& \text { where } \alpha=v F A w\left(C^{*}-C_{\text {cutoff }}\right) \text { and } \beta=\frac{\pi \sqrt{D}}{w} .
\end{aligned}
$$

The unit of $\alpha$ is coulomb (charge) and that of $\beta^{2}$ is second ${ }^{-1}$. Intuitively, $\alpha$ is the battery capacity, and $\beta$ is the measure of battery nonlinearity. Note that $\alpha$ increases as $A w$ (i.e. the battery size) increases. Also, $\beta$ decreases as $D$ decreases, i.e. diffusion limitations worsen. A larger value of $\beta$ indicates a better battery. Indeed, if $\beta$ is sufficiently large, the second term of the equation (2) becomes negligible, and we obtain the model of an ideal power source.

Under constant-current discharge, $i(t)=I$, the equation (2) reduces to the following form:

$$
\alpha=I\left[L+2 \sum_{m=1}^{\infty} \frac{1-e^{-\beta^{2} m^{2} L}}{\beta^{2} m^{2}}\right] .
$$

For a general case, we approximate the time-varying discharge current $i(t)$ by $n$-step piece-wise constant load: Figure 1 shows an example of such an approximation. After substitution of $i(t)$ into (2) and integrating the sum term by term (the series is absolutely convergent), we obtain

$$
\begin{gathered}
\alpha \approx \sum_{k=0}^{n-1} I_{k} F\left(L, t_{k}, t_{k+1}, \beta\right), \text { where } \\
F\left(L, t_{k}, t_{k+1}, \beta\right)=t_{k+1}-t_{k}+2 \sum_{m=1}^{\infty} \frac{e^{-\beta^{2} m^{2}\left(L-t_{k+1}\right)}-e^{-\beta^{2} m^{2}\left(L-t_{k}\right)}}{\beta^{2} m^{2}} .
\end{gathered}
$$

Note that $t_{0}=0$ and $t_{n}=L$. For $n=1$, (4) reduces to the special case (3). The magnitude of the series terms in (3) diminish very rapidly as $m$ grows. As our experimental results indicate, employing only the first 10 terms already yields quite accurate lifetime predictions $^{1}$.

In comparison to [13], the battery model described by the equation 4 is better in terms of accuracy and computational complexity. Unlike the model in [13], it does not involve square roots and does not require an approximation of the complementary error function (see [13] for details). However, note that the both models produce the same results numerically.

Parameter Estimation: Before one can use the proposed model, the quantities $\alpha$ and $\beta$ need to be estimated from profiling data for the modeled battery. Simple experiments with constant discharge rates are sufficient. Therefore, one can utilize (3) for estimation purposes.

Under a given constant load, the battery voltage drops from the open-circuit value $V_{o}$ to some cutoff value $V_{\text {cutoff }}$ over time. Let

\footnotetext{
${ }^{1}$ Our calculations involving 100000 terms have resulted in negligible improvement in accuracy, while considerably slowing down the lifetime computation process.
}

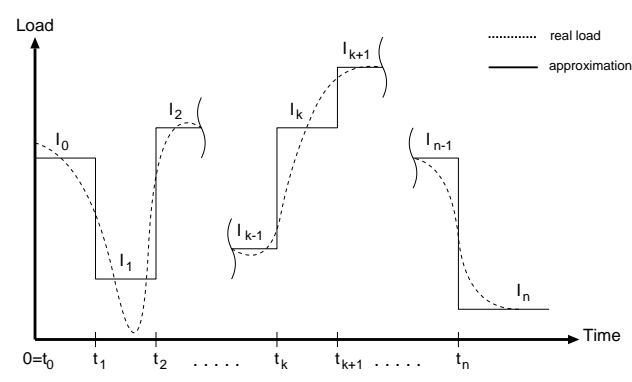

Figure 1: $n$-Step Staircase Approximation of Variable Load.

the observed lifetime be the time when the battery voltage reaches $V_{\text {cutoff }}$, and let the predicted lifetime be the earliest time instance for which the equality (3) holds. Since (3) is hard to solve for the unknown $L$, we fit the load values $I$ instead, for a given set of observed lifetimes: $I=\alpha /\left[L+2 \sum_{m=1}^{10} \frac{1-e^{-\beta^{2} m^{2} L}}{\beta^{2} m^{2}}\right]$.

The objective is to find $\alpha$ and $\beta$ such that $I$ matches actual load values as closely as possible. The fit can be obtained using leastsquares estimation.

Lifetime Computation: Given the $n$-step staircase description of the load profile, we want to determine the failing step at which the battery becomes exhausted. Since a profile may contain rest periods, early failures may be masked after recovery; therefore, it is necessary to find the earliest failing step. For such step $u$, lifetime $L \in\left[t_{u}, t_{u+1}\right]$ is equal to the root $x_{0}$ of the following equation:

$$
\alpha-\sum_{k=0}^{u-1} I_{k} F\left(x, t_{k}, t_{k+1}, \beta\right)-I_{u} F\left(x, t_{u}, x, \beta\right)=0 .
$$

For a given step under consideration, we use the modified secant method to solve (5). Since we must find the earliest step $u$, we examine load profile steps $\{0,1, \ldots, u, \ldots, n-2, n-1\}$ in increasing order. Once the root is detected, it is returned as the estimated lifetime $L$.

\section{SIMULATED BATTERY}

The quality of our model is evaluated with respect to the results produced by the low-level simulator DUALFOIL [4, 7]. DUALFOIL numerically simulates a set of partial differential equations governing the behavior of a rechargeable lithium-ion cell. Over 50 parameters must be supplied in order to specify a simulated battery. Studies $[6,1]$ have already demonstrated the high quality of DUALFOIL by comparing simulation and measurement results. Here, we describe our simulation settings and discuss similarities and differences between our simulated battery and a real battery used in the Itsy pocket computer $[15,16]$.

\subsection{Itsy Battery}

Itsy is powered by a rechargeable $2.2 \mathrm{~W}$-hour lithium-ion battery with an open-circuit voltage of $4.1 \mathrm{~V}$ and nominal discharge rate of $640 \mathrm{~mA}$. We used the results of five constant-load experiments from [16]: the Itsy battery was discharged to $3.0 \mathrm{~V}$ directly by an electronic load operating in a constant current mode. The discharge rates were set to $1 C, 1.5 C, 0.2 C, 0.1 C$, and $0.05 C$, respectively called $1^{*}-\mathrm{T} 5^{*}$, where $C$ is $640 \mathrm{~mA}$.

Parameter estimations based on tests $\mathrm{T} 1^{*}-\mathrm{T} 5^{*}$ produce $\alpha=35220$ and $\beta=0.637$. Figure 2 shows that our model fits the measurement data very well: the maximum error is less than $1 \%$. However, this result can be somewhat misleading. Figure 2 also shows the fit of Peukert's model $a=I L^{b}[9]$ and the ideal source model $c=I L$ (i.e. the delivered charge is proportional to the load $)^{2}$. The ideal source model fitted the data within $16 \%$ error margin, which suggests that the Itsy battery behavior does not exhibit significant nonlinear effects. Peukert's law produced estimates with the maximum error

\footnotetext{
${ }^{2} I$ is the current, $L$ is the lifetime, and $a, b, c$ are model coefficients.
} 


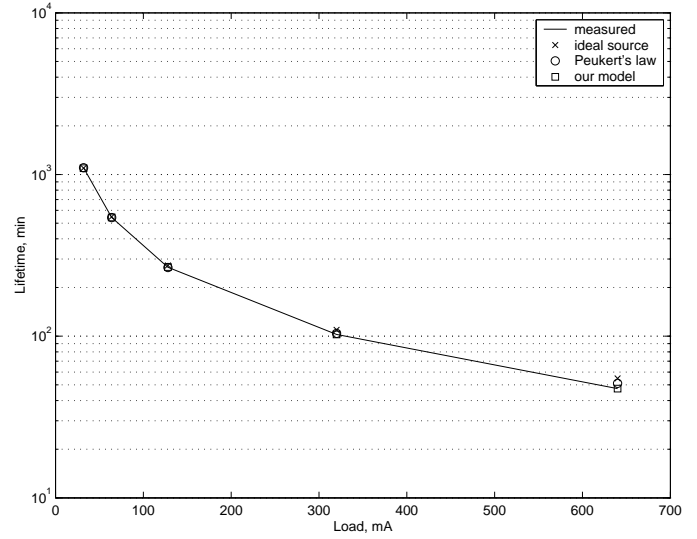

Figure 2: Predicted and Measured Lifetimes for Constant Loads.

of $8 \%$ : it is better than the ideal source model, but worse than our model. Since the Itsy battery is relatively well-behaved, predicting its lifetime within an acceptable error margin is not difficult. In other words, our model is not exercised in terms of how well it can account for nonlinear effects. The nonlinear component of our model (4) is the term involving $\beta$ : if $\beta$ is sufficiently large, the nonlinear term becomes negligible. We want to test our model against a battery with low $\beta$. Our simulated battery provides this opportunity.

\subsection{DUALFOIL Battery}

Given over 50 default DUALFOIL settings, we adjusted 30 simulation parameters, based on the data from [1]. (Our modified settings are omitted in this paper due to the lack of space.) To obtain lifetimes, we must also specify the cutoff voltage. To make a proper choice we used lifetime measurements for the Itsy pocket computer $[15,16]$, described next.

From $[15,16]$, we selected ten cases of Itsy running with audio output enabled (T1-T10) and ten similar cases but with audio output disabled (T11-T20). The lifetimes ranged from 2 hours to 12 days, and the average battery power $P_{\text {average }}$ ranged from $8 \mathrm{~mW}$ to $825 \mathrm{~mW}$. To obtain current consumption data, we first assumed that the battery voltage was constant and equal to $V_{\text {average }}=3.75 \mathrm{~V} \mathrm{[15]}$; then, we divided the reported $P_{\text {average }}$ by $V_{\text {average }}$. These loads are summarized in Table 1.

\begin{tabular}{|l|l|l|c|}
\hline Test & Name & Description & $I_{\text {average }}, \mathrm{mA}$ \\
\hline \hline T1 & MPEG & video, 206MHz & 222.7 \\
\hline T2 & Dictation & speech input, 206Hz & 204.5 \\
\hline T3 & Talk1 & speech output, 206MHz & 108.3 \\
\hline T4 & Talk2 & speech output, 74MHz & 107.5 \\
\hline T5 & Talk3 & speech output, 74MHz (LV) & 94.9 \\
\hline T6 & WAV1 & audio, 206MHz & 84.3 \\
\hline T7 & WAV2 & audio, 59MHz & 75.5 \\
\hline T8 & Idle1 & operating system, 206MHz & 28.0 \\
\hline T9 & Idle2 & operating system, 59MHz & 19.5 \\
\hline T10 & SleepDC & sleep with a daughter-card & 3.0 \\
\hline \hline T11 & MPEG-AOD & MPEG, audio output disabled & 188.3 \\
\hline T12 & Dictation-AOD & Dictation, audio output disabled & 203.7 \\
\hline T13 & Talk1-AOD & Talk1, audio output disabled & 100.5 \\
\hline T14 & Talk2-AOD & Talk2, audio output disabled & 100.0 \\
\hline T15 & Talk3-AOD & Talk3, audio output disabled & 87.5 \\
\hline T16 & WAV1-AOD & WAV1, audio output disabled & 49.6 \\
\hline T17 & WAV2-AOD & WAV2, audio output disabled & 41.3 \\
\hline T18 & Idle1-AOD & Idle1, audio output disabled & 28.3 \\
\hline T19 & Idle2-AOD & Idle2, audio output disabled & 19.7 \\
\hline T20 & Sleep-AOD & sleep, audio output disabled & 2.0 \\
\hline
\end{tabular}

Table 1: Description of Constant Loads for DUALFOIL.

\begin{tabular}{|c|c|c||c|c|c|}
\hline Test & Description & $I_{\text {average }}, \mathrm{mA}$ & Test & Description & $I_{\text {average }}, \mathrm{mA}$ \\
\hline \hline T21 & IAT & 628.0 & T27 & DSD & 234.1 \\
\hline T22 & IAR & 494.7 & T28 & TSD & 137.9 \\
\hline T23 & IST & 425.6 & T29 & WSD & 113.9 \\
\hline T24 & ISR & 292.3 & T30 & ISD & 57.6 \\
\hline T25 & IAD & 265.6 & T31 & SSD & 32.5 \\
\hline T26 & MSD & 252.3 & T32 & Boot & 300.0 \\
\hline
\end{tabular}

Table 2: DUALFOIL Loads Assuming Additional Peripherals.

For the simulated battery, the open-circuit voltage is $V_{o}=4.3 \mathrm{~V}$, and we set the cutoff voltage to $V_{\text {cutoff }}=3.2 \mathrm{~V}$. (Note that the average of $V_{o}$ and $V_{\text {cutoff }}$ is $3.75 \mathrm{~V}$, the same as reported in [15].) The resulting DUALFOIL simulation data and measured data are displayed in Figure 3, which also includes the lifetimes for tests $\mathrm{T}^{*}-\mathrm{T} 5^{*}$. For the currents ranging from $2 \mathrm{~mA}$ to $200 \mathrm{~mA}$, the maximum lifetime error was only $4 \%$. These results indicate that our battery simulation settings are realistic. However, under high loads (tests $\mathrm{T}^{*}$ and $\mathrm{T} 2^{*}$ ) the simulated battery behaves much worse than the Itsy battery, suggesting that the DUALFOIL battery is highly nonlinear. The simulated battery, although inspired by the Itsy battery, is not intended to represent it, as we know neither chemical nor mechanical structure of the latter.

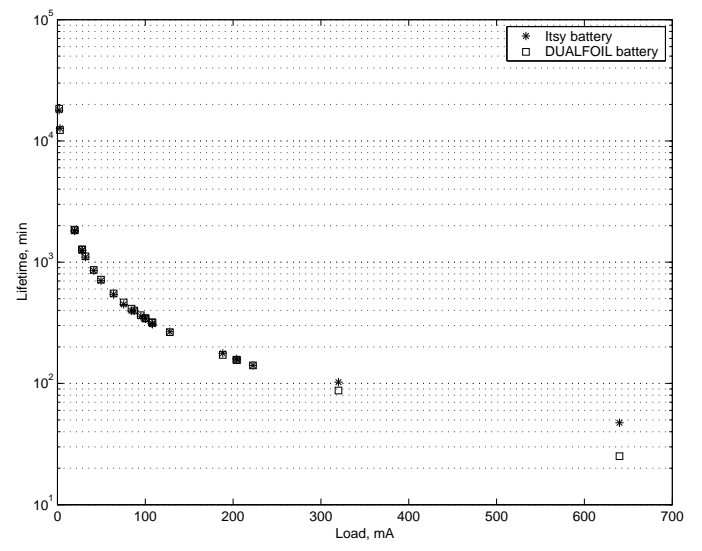

Figure 3: Simulated (DUALFOIL) and Measured (Itsy) Lifetimes.

The next step is to estimate the parameters of our model so that it can represent the simulated battery. We simulated twelve additional constant load tests assuming that Itsy had additional peripherals: a Microdrive hard disk and a WaveLAN wireless card $[15]^{3}$. The additional tests are summarized in Table 2. Each test is described by a 3-letter code, except for the last one, Boot, which corresponds to rebooting Itsy (it is assumed that the current drawn in this case is approximately $300 \mathrm{~mA}$ ). The letter abbreviations used are as follows: (1) Itsy: I - Idle1, M - MPEG, D - Dictation, T - Talk1, W WAV1, S - SleepDC; (2) Microdrive: S - standby, A - access; (3) WaveLAN: D - doze, $\mathrm{R}$ - receive, $\mathrm{T}$ - transmit. In the 3-letter code, the first, the second, and the third letters indicate the state of Itsy, the Microdrive, and the WaveLAN, respectively. For example, IAT (test T21) means that Itsy is idle, the hard disk is being accessed, and the wireless card is transmitting.

Given the battery simulation settings and the complete set of 32 constant load tests T1-T32, we obtained a wide range of lifetimes: from $1 / 2$ hour to 300 hours. Based on these results, the estimated parameters for our model are: $\alpha=40027$ and $\beta=0.276$. The lower value for $\beta$ means that the simulated battery exhibits stronger non-

\footnotetext{
${ }^{3}$ The power consumption of the Microdrive is $66 \mathrm{~mW}$ in standby mode and $825 \mathrm{~mW}$ while accessed. The WaveLAN consumes $45 \mathrm{~mW}$ in the doze state, $925 \mathrm{~mW}$ in reception mode, and $1425 \mathrm{~mW}$ while transmitting.
} 
linearity (thus, creating a greater challenge for our model), than the Itsy battery.

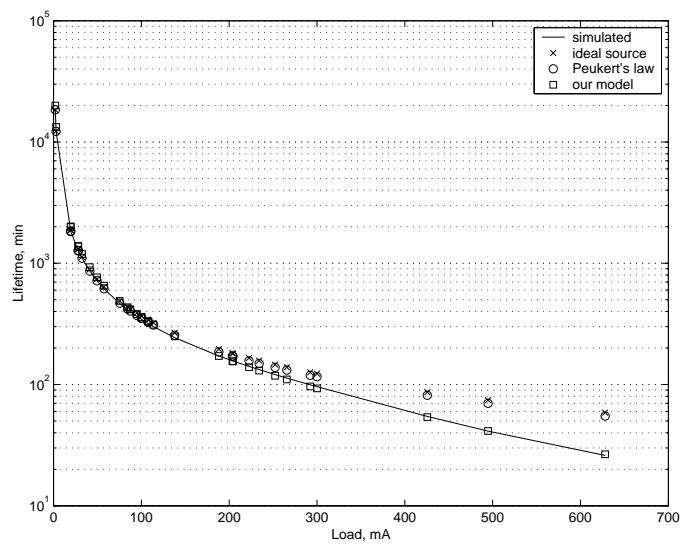

Figure 4: Predicted and Simulated Lifetimes for Constant Loads.

Prediction accuracy of our model was within $10 \%$. The model fit is shown in Figure 4. For comparison purposes, Figure 4 also displays the fits due to the Peukert's law and the ideal source model. One can see that neither of the two could adequately handle strong nonlinearity: the maximum error exceeded $100 \%$.

\section{VARIABLE LOAD SIMULATIONS}

In this section we describe twenty two variable-current experiments performed with the DUALFOIL simulator and compare the simulated lifetimes with the estimates based on our model. We assume that Itsy is powered by the DUALFOIL battery, and we investigate how Itsy power profile variations affect lifetimes. Recall that for the simulated battery $\alpha=40027$ and $\beta=0.276$. To highlight the nonlinearity of the DUALFOIL battery, we also provide predictions for the real Itsy battery, with $\alpha=35220$ and $\beta=0.637$.

In our experiments, we use loads with coarse-grain timing $(0.1$ minute timing scale resolution). During each step $k$ (see Figure 1), the discharge current $I_{k}$ is assumed to equal some averaged constant. Note that specifying timing in fractions of a minute is specific only to the experimental results, not the proposed model itself: a user can use any time scale. In other words, timing granularity is a user's choice. Lower timing granularity yields more accurate load description. At the extreme, one may specify the battery current at each clock cycle, if desired. According to [10], however, timing scale resolution of less than 1 second is unnecessary. Also, the battery lifetime is normally in the order of tens and hundreds of minutes. Intensive computations using the accuracy of 1 second to predict the battery lifetime of several hours may often be wasteful, since battery parameters fluctuate due to battery manufacturing lot variations, discharge-recharge cycling effects, and temperature changes. Here, we predict lifetimes with the accuracy of 0.1 minute.

\subsection{Averaging Battery Voltage}

Before we present our simulation results, we address the issue of battery voltage averaging. Note that our battery model does not involve battery voltages. A user must specify the current drawn from the battery during application execution. If the battery current $i(t)$ is not known, a user can assume the average battery voltage $V_{\text {average }}$, and let $i(t)=P(t) /\left(\mu V_{\text {average }}\right)$, where $\mu$ is the efficiency of the DC-DC converter and $P(t)$ is the power consumption of an application. Is the assumption of the constant average battery voltage acceptable? To answer this question, we use the measurement data from [16], presented in Table 3. The first column shows different types of Itsy applications, and the second column shows the corresponding average battery power $P_{\text {average }}$, consumed while the Itsy battery was able to maintain the system on-line. In the next set of experiments, the battery was directly connected to an electronic

\begin{tabular}{|c|c|c|c|c|c|}
\hline \multirow[t]{2}{*}{ Name } & \multirow{2}{*}{$\begin{array}{c}P_{\text {average }} \\
\mathrm{mW}\end{array}$} & \multirow{2}{*}{$\begin{array}{l}\text { Constant Power } \\
\text { Lifetime, min }\end{array}$} & \multirow{2}{*}{$\begin{array}{l}\text { Constant } \text { Current } \\
\text { Lifetime, } \min \\
\end{array}$} & \multicolumn{2}{|c|}{ Error } \\
\hline & & & & $\Delta \%$ & $\Delta \min$ \\
\hline MPEG & 835 & $\overline{152.4}$ & 150.6 & 1.2 & $\overline{1.8}$ \\
\hline Dictation & 767 & 167.4 & 168.0 & 0.4 & 0.6 \\
\hline Talk1 & 406 & 327.0 & 321.0 & 1.8 & 6.0 \\
\hline Talk2 & 403 & 330.6 & 324.0 & 2.0 & 6.6 \\
\hline Talk3 & 356 & 376.8 & 370.8 & 1.6 & 6.0 \\
\hline WAV1 & 316 & 421.8 & 415.8 & 1.4 & 6.0 \\
\hline WAV2 & 283 & 473.4 & 467.4 & 1.3 & 6.0 \\
\hline Idle1 & 105 & 1309.8 & 1284.0 & 2.0 & 25.8 \\
\hline Idle2 & 73 & 1888.8 & 1839.6 & 2.6 & 49.2 \\
\hline
\end{tabular}

Table 3: Effect of Battery Voltage Averaging.

\begin{tabular}{|l|l|l|l|}
\hline Case & Description & Load Values $S_{I}, \mathrm{~mA}$ & Load Timing $S_{t}, \mathrm{~min}$ \\
\hline \hline $\mathrm{C} 1$ & IAT-off-IAT & $(628.0,0,628.0)$ & $(0,19.5,26.0)$ \\
\hline C2 & IAR-off-IAR & $(494.7,0,494.7)$ & $(0,31.0,41.3)$ \\
\hline C3 & IST-off-IST & $(425.6,0,425.6)$ & $(0,41.0,54.6)$ \\
\hline C4 & ISR-off-ISR & $(292.3,0,292.3)$ & $(0,74.6,99.5)$ \\
\hline C5 & MPEG-off-MPEG & $(222.7,0,222.7)$ & $(0,105.7,140.9)$ \\
\hline \hline C6 & IAT-off-IAT & $(628.0,0,628.0)$ & $(0,19.5,29.9)$ \\
\hline C7 & IAT-off-IAT & $(628.0,0,628.0)$ & $(0,19.5,22.1)$ \\
\hline C8 & IAT-off-IAT & $(628.0,0,628.0)$ & $(0,23.4,29.9)$ \\
\hline C9 & IAT-off-IAT & $(628.0,0,628.0)$ & $(0,15.6,22.1)$ \\
\hline \hline C20 & {$\left[\right.$ IAR-IAT ${ }^{\infty}$} & $\left([494.7,628.0]^{\infty}\right)$ & $\left([0,1.0]_{2.0}^{\infty}\right)$ \\
\hline C21 & {$[\text { IAR-IAT-ISD }]^{\infty}$} & $\left([494.7,628.0,57.6]^{\infty}\right)$ & $\left([0,1.0,2.0]_{3.0}^{\infty}\right)$ \\
\hline C22 & $5.0+5.0 / m i n$ & $(5.0,10.0,15.0, \ldots)$ & $(0,1.0,2.0, \ldots)$ \\
\hline
\end{tabular}

Table 4: Interrupted, Periodic, and Linear Load Profiles.

load. In the first set of experiments, the electronic load operated in a constant power mode, and in the second set of experiments, it operated in a constant current mode. The constant power was set to $P_{\text {average }}$ and the constant current was set to $P_{\text {average }} / V_{\text {average }}$, where $V_{\text {average }}$ was $3.75 \mathrm{~V}$. The corresponding lifetimes are reported in the third and fourth columns. Note that during constant-power discharge, the battery voltage is decreasing, and thus the battery current is increasing; on the other hand, during constant-current discharge the battery voltage is decreasing, but the battery current is constant. Under our assumption of the constant battery voltage, the constant-current discharge and constant-power discharge should be equivalent. Indeed, the lifetimes in the third and fourth columns are very close: the relative error does not exceed $3 \%$. These results demonstrate that the accuracy loss due to battery voltage averaging is often acceptable.

\subsection{Variable-Load Lifetimes}

Twenty two variable load tests are summarized in Tables 4 and 5. To specify a staircase description of the load profile, as shown in Figure 1, we introduce two ordered sets: the set of load values $S_{I}=$ $\left(I_{0}, I_{1}, \ldots, I_{n-1}\right)$ and the set of load start times $S_{t}=\left(t_{0}, t_{1}, \ldots, t_{n-1}\right)$. Consider case C1, for example, with $S_{I}=(628.0,0,628.0)$ and $S_{t}=$ $(0,19.5,26.0)$. This means that the discharge current is $628.0 \mathrm{~mA}$ in the intervals $[0,19.5)$ and $[26.0, \infty)$, while there is no load in the interval $[19.5,26.0)$. For periodic loads, we provide a single specification for the first period only. The description of this period is enclosed in the square brackets. The superscript indicates the number of periods, and the subscript indicates the period duration. In case $\mathrm{C} 19, S_{I}=\left([75.5,94.9,204.5,222.7]^{10}, 222.7\right)$ and $S_{t}=\left([0,5.0,10.0,15.0]_{20.0}^{10}, 200.0\right)$. This means that the battery was subject to 10 periods of length 20 minutes each, before the constant load of $222.7 \mathrm{~mA}$ was applied. Within a period, $75.5 \mathrm{~mA}$ was drawn for the first 5 minutes, $94.9 \mathrm{~mA}$ for the second $5 \mathrm{~min}$ utes, $204.5 \mathrm{~mA}$ for the next 5 minutes, and $222.7 \mathrm{~mA}$ for the last 5 minutes. Note that the last test $\mathrm{C} 22$ is a linear load increasing from $5 \mathrm{~mA}$ by $5 \mathrm{~mA}$ every minute.

Table 6 gives simulated and predicted lifetimes for the 22 variable load tests performed. Our model (with $\alpha=40027$ and $\beta=$ 0.276 ) was accurate within 5\% error margins, with a maximum absolute error of less than 4 minutes. These results demonstrate that 


\begin{tabular}{|c|c|c|c|}
\hline Case & Description & Load Values $S_{I}, \mathrm{~mA}$ & Load Timing $S_{t}$, min \\
\hline$\overline{\mathrm{C} 10}$ & "Boot-IAT-IAR-MSD-DSD-TSD-WSD-IAD & 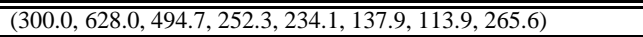 & $\overline{(0,0.5,5.5,10.5,35.5,60.5,85.5,110.5)}$ \\
\hline $\mathrm{C} 11$ & Boot-WSD-TSD-DSD-MSD-IAR-IAT-IAD & $(300.0,113.9,137.9,234.1,252.3,494.7,628.0,265.6)$ & $(0,0.5,25.5,50.5,75.5,, 100.5,105.5,110.5)$ \\
\hline $\mathrm{C} 12$ & Boot-WSD-TSD-DSD-MSD-IAR-off-Boot-IAT-IAD & $(300.0,113.9,137.9,234.1,252.3,494.7,0,300.0,628.0,265.6)$ & $(0,0.5,25.5,50.5,75.5,100.5,105.5,130.5,131.0,136.0)$ \\
\hline $\mathrm{C} 13$ & Boot-[IAT-IAR-MSD-DSD-TSD-WSD] $]^{5}$-IAD & $\left(300.0,[628.0,494.7,252.3,234.1,137.9,113.9]^{5}, 265.6\right)$ & $\left(0,[0.5,1.5,2.5,7.5,12.5,17.5]_{22.5}^{5}, 110.5\right)$ \\
\hline $\mathrm{C} 14$ & Boot-[WSD-TSD-DSD-MSD-IAR-IAT] ${ }^{5}$-IAD & $\left(300.0,[113.9,137.9,234.1,252.3,494.7,628.0]^{5}, 265.6\right)$ & $\left(0,[0.5,5.5,10.5,15.5,20.5,21.5]_{22.5}^{5}, 110.5\right)$ \\
\hline C15 & "MPEG-Dictation-Talk1-WAV1-MPEG & $\overline{(222.7,204.5,108.3,84.3,222.7)}$ & $\overline{(0,50.0,100.0,150.0,200.0)}$ \\
\hline C16 & WAV1-Talk1-Dictation-MPEG-MPEG & $(84.3,108.3,204.5,222.7,222.7)$ & $(0,50.0,100.0,150.0,200.0)$ \\
\hline $\mathrm{C} 17$ & WAV1-Talk1-Dictation-off-MPEG-MPEG & $(84.3,108.3,204.5,0,222.7,222.7)$ & $(0,50.0,100.0,150.0,200.0,250.0)$ \\
\hline $\mathrm{C} 18$ & [WAV1-Talk1-Dictation-MPEG] ${ }^{10}$-MPEG & $\left([84.3,108.3,204.5,222.7]^{10}, 222.7\right)$ & $\left([0,5.0,10.0,15.0]_{20.0}^{10}, 200.0\right)$ \\
\hline $\mathrm{C} 19$ & [WAV2-Talk3-Dictation-MPEG] ${ }^{10}$-MPEG & $\left([75.5,94.9,204.5,222.7]^{10}, 222.7\right)$ & $\left([0,5.0,10.0,15.0]_{20.0}^{10}, 200.0\right)$ \\
\hline
\end{tabular}

Table 5: Non-Periodic Load Profiles.

\begin{tabular}{|c|c|c|c|c|c|}
\hline \multirow[b]{2}{*}{ Case } & \multicolumn{4}{|c|}{ DUALFOIL Battery } & \multirow{2}{*}{$\begin{array}{c}\text { Itsy Battery } \\
\begin{array}{c}\text { Predicted } \\
\text { min }\end{array}\end{array}$} \\
\hline & $\begin{array}{l}\text { Simulated } \\
\text { min }\end{array}$ & $\min$ & \multicolumn{2}{|c|}{ Predicted } & \\
\hline $\mathrm{C} 1$ & 36.4 & 36.2 & 0.5 & 0.2 & 55.0 \\
\hline $\mathrm{C} 2$ & 57.2 & 55.8 & 2.4 & 1.4 & 73.9 \\
\hline C3 & 74.2 & 71.8 & 3.2 & 2.4 & 88.8 \\
\hline $\mathrm{C} 4$ & 128.1 & 124.9 & 2.5 & 3.2 & 137.8 \\
\hline $\mathrm{C} 5$ & 178.5 & 176.7 & 1.0 & 1.8 & 185.8 \\
\hline$\overline{\mathrm{C} 6}$ & $\overline{41.5}$ & $\overline{441.0}$ & $\overline{1.2}$ & $\overline{0.5}$ & $\overline{58.9}$ \\
\hline C7 & 30.6 & 30.8 & 0.7 & 0.2 & 51.1 \\
\hline $\mathrm{C} 8$ & 37.0 & 37.4 & 1.1 & 0.4 & 55.0 \\
\hline C9 & 35.4 & 35.2 & 0.6 & 0.2 & 55.0 \\
\hline$\overline{\mathrm{C} 10}$ & $\overline{135.2}$ & 132.6 & $\overline{c 1.9}$ & $\overline{2.6}$ & $\overline{144.3}$ \\
\hline C11 & 108.8 & 107.4 & 1.3 & 1.4 & 144.3 \\
\hline $\mathrm{C} 12$ & 159.0 & 155.4 & 2.3 & 3.6 & 169.3 \\
\hline $\mathrm{C} 13$ & 133.8 & 131.7 & 1.6 & 2.1 & 144.3 \\
\hline C14 & 132.9 & 129.7 & 2.4 & 3.2 & 144.3 \\
\hline C15 & 207.6 & 209.2 & 0.8 & 1.6 & 211.4 \\
\hline C16 & 202.4 & 200.7 & 0.8 & 1.7 & 211.4 \\
\hline C17 & 253.8 & 251.2 & 1.0 & 2.6 & 261.4 \\
\hline C18 & 204.6 & 204.6 & 0.0 & $\overline{0.0}$ & 211.4 \\
\hline C19 & 209.4 & 208.7 & 0.3 & 0.7 & 216.4 \\
\hline $\mathrm{C} 20$ & $\overline{31.7}$ & $\overline{33.2}$ & $\overline{4.7}$ & $\overline{1.5}$ & $\overline{\overline{55.3}}$ \\
\hline $\mathrm{C} 21$ & 55.9 & 55.9 & 0.0 & $\overline{0.0}$ & 79.6 \\
\hline C22 & 97.5 & 94.5 & 3.1 & 3.0 & 112.2 \\
\hline
\end{tabular}

Table 6: Predicted and Simulated Lifetimes for Variable Loads.

our model matches the simulation-based approach in its generality and accuracy. In the last column of Table 6 , we also show predicted lifetimes for the Itsy battery, computed by our model with $\alpha=35220$ and $\beta=0.637$. Note that the entries in the last column (our predictions for the Itsy battery) and the entries in the second column and the third columns (simulated and predicted lifetimes of the DUALFOIL battery) correspond to two different batteries.

\subsection{Nonlinear Effects}

Here, we outline several interesting phenomena observed in our variable load experiments with the DUALFOIL battery. We also discuss our predictions for the Itsy battery, presented in the last column of Table 6.

Recovery effect: Let $L_{\text {original }}$ denote the lifetime of the original profile with no load relaxation exercised. Assume that a rest period or an idle load of duration $\delta$ is inserted into the profile. Let $L_{\text {unaffected }}$ denote the expected lifetime as if no recovery takes place: $L_{\text {unaffected }}=L_{\text {original }}+\delta$. Otherwise, the lifetime $L$ should be greater than $L_{\text {unaffected }}$. The quantity $\frac{L-L_{\text {unaffected }}}{L_{\text {original }}} 100 \%$ is the lifetime extension due to the recovery effect. Table 7 reports this data for the DUALFOIL battery under cases C1-C9. The first five cases $\mathrm{C} 1, \mathrm{C} 2, \mathrm{C} 3, \mathrm{C} 4$, and $\mathrm{C} 5$ are based on the original constant load tests T21, T22, T23, T24, and T1, respectively. In each case, the battery is discharged at the constant rate, then the load is turned off for a while, and afterwards discharging is continued at the same rate. The start time and duration $\delta$ of the rest period is $0.75 L_{\text {original }}$ and $0.25 L_{\text {original }}$, respectively. For example, in case $\mathrm{C} 1, L_{\text {original }}$ is equal to 26.0 minutes (the lifetime in T21); therefore, the rest pe-

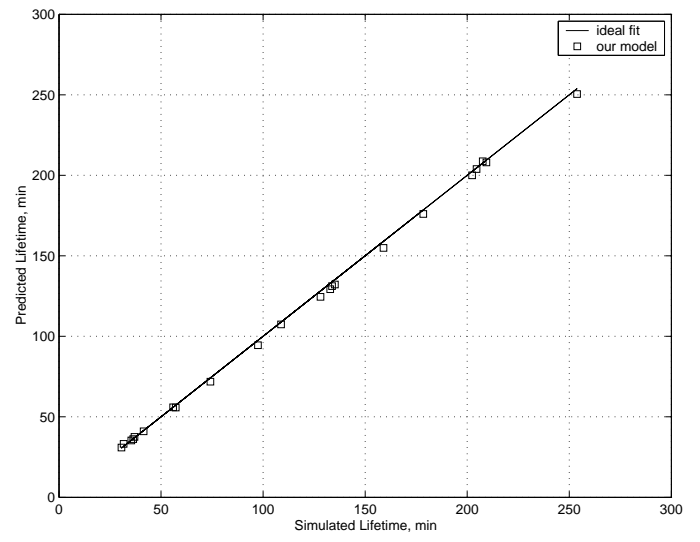

Figure 5: Predicted vs. Simulated Lifetimes for Variable Loads.

riod started after 19.5 minutes elapsed and lasted for 6.5 minutes, which resulted in the $15 \%$ lifetime improvement. In the next four cases C6-C9, we used $\mathrm{C} 1$ as a basis. In $\mathrm{C} 6$ (C7), the duration of the rest period was increased (decreased) by $0.15 L_{\text {original }}$. In C8 (C9), the start time of the rest period was increased (decreased) by $0.15 L_{\text {original }}$. These nine cases indicate that (1) the greater the load, the greater the recovery effect, (2) the longer the rest period, the greater the recovery effect, and (3) the greater the depth of discharge, the greater the recovery effect.

For heavy periodic loads such as C20, the battery can deliver extra charge provided that an idle load is inserted within each period. In case $\mathrm{C} 20$, the period was 2 minutes, and in case $\mathrm{C} 21$, the period was extended by 1 minute to accommodate the idle load. Note that this idle mode invokes not only a delay penalty but also an energy penalty because the system is not off-line. Nevertheless, in case C21 the battery serviced 18 full periods; while, in case C20 it serviced only 15 full periods.

For cases $(\mathrm{C} 1, \mathrm{C} 2, \mathrm{C} 3, \mathrm{C} 4, \mathrm{C} 5)$, our model for the Itsy battery predicts $L_{\text {original }}=(48.5,63.6,75.1,109.8,150.5)$ minutes, respectively. Note that $\delta=(6.5,10.3,13.6,24.9,35.2)$ minutes, yielding $L_{\text {unaffected }}=(55.0,73.9,88.7,134.7,185.7)$. By comparing $L_{\text {unaffected }}$ to the corresponding entries in the last column of Table 6, one can see that predicted recovery is negligible. Also for cases (C6, C7, C8, C9), with $L_{\text {original }}=(48.5,48.5,48.5,48.5)$, $\delta=(10.4,2.6,6.5,6.5)$, and $L_{\text {unaffected }}=(58.9,51.1,55.0,55.0)$, respectively, our predictions indicate that there will be no recovery observed. (For verification purposes, C1, C2, and C6 were actually tested on the Itsy battery, and indeed showed negligible recoveries of under 1\%, which confirms our predictions.) For cases C20 and $\mathrm{C} 21$, our model predicts the lifetimes of 55.3 minutes ( 27 full periods) and 79.6 minutes (26 full periods), respectively. Since there is no recovery in $\mathrm{C} 21$, the energy penalty due to the idle load is not compensated, which results in the slightly worse lifetime than in case C20. (Actual experiments C20 and C21 showed 29 and 28 full periods, respectively, for the Itsy battery). 


\begin{tabular}{|l|cc|c|c|}
\hline Case & $L_{\text {original }}, \mathrm{min}$ & $L_{\text {unaffected }}, \mathrm{min}$ & $L, \mathrm{~min}$ & Recovery, $\%$ \\
\hline \hline $\mathrm{C} 1$ & 26.0 & 32.5 & 36.4 & 15.0 \\
\hline C2 & 41.3 & 51.6 & 57.2 & 13.6 \\
\hline C3 & 54.6 & 68.2 & 74.2 & 11.0 \\
\hline C4 & 99.5 & 124.4 & 128.1 & 3.7 \\
\hline C5 & 140.9 & 176.1 & 178.5 & 1.7 \\
\hline \hline C6 & 26.0 & 36.4 & 41.5 & 19.6 \\
\hline C7 & 26.0 & 28.6 & 30.6 & 7.7 \\
\hline C8 & 26.0 & 32.5 & 37.0 & 17.3 \\
\hline C9 & 26.0 & 32.5 & 35.4 & 11.2 \\
\hline
\end{tabular}

Table 7: DUALFOIL Battery Recovery under Interrupted Loads.

Heavy Loading: As cases C10-C14 demonstrate, when the profile contains high-current loads, different profile shapes may result in significantly different lifetimes. Note that in these cases we sequence the loads in various orders, without changing the peak or average power of the resulting profile. The peak load is $628.0 \mathrm{~mA}$. The first (Boot) and the last (IAD) loads are not affected by profile changes in all of the cases in question.

Cases C10 and C11 suggest that it is beneficial to sequence nonperiodic loads in non-increasing order of their current consumption values. The non-increasing ordering in $\mathrm{C} 10$ (except for the first and the last loads) forced high-current loads to be serviced early, when the battery was relatively "young". As a result, the battery lasted for almost 25 minutes after the last load started. On the contrary, the non-decreasing ordering in $\mathrm{C} 11$ led to a failure before the last load could start.

Rest periods are especially helpful when the discharge profile contains high currents, as case $\mathrm{C} 12$ indicates. In $\mathrm{C} 12$, the failing profile of $\mathrm{C} 11$ was interrupted after 105.5 minutes, and a 25 -minute long rest period was inserted. It was followed by a 30 -second reboot, yielding a charge penalty of $150 \mathrm{~mA}-\mathrm{min}$. The total delay penalty was 25.5 minutes. Excluding this delay, the battery lasted for 133.5 minutes, which is a significant improvement over 108.8 minutes in $\mathrm{C} 11$.

The last two cases $\mathrm{C} 13$ and $\mathrm{C} 14$ are periodic versions of $\mathrm{C} 10$ and $\mathrm{C} 11$, respectively. Since high-current and low-current loads became distributed across the profile, the lifetimes were not very different. One can observe that the battery lasted longer in case C14 than in case C11, which suggests that preempting and looping might relieve the negative impact of the non-decreasing load ordering.

However, all of the above effects are not predicted for the Itsy battery. Note that the lifetimes in cases $\mathrm{C} 10, \mathrm{C} 11, \mathrm{C} 13$, and $\mathrm{C} 14$ are the same, equal to 144.3 minutes. In $\mathrm{C} 12$, the delay penalty of 25.5 minutes would yield the lifetime of 169.8 minutes, which is slightly better than 169.3 minutes predicted by our model. This marginal loss is due to the energy penalty of a reboot.

Light Loading: Cases C15-C19 are different permutations of light loads: the peak current is $222.7 \mathrm{~mA}$. Consequently, the battery lifetime does not significantly depend on the load profile shape, unlike cases $\mathrm{C} 10-\mathrm{C} 14$. Indeed, the lifetimes in $\mathrm{C} 15$ and $\mathrm{C} 16$ are not very different, despite the fact that in $\mathrm{C} 15$ (C16) the loads, except for the last one, are scheduled in non-increasing (non-decreasing) order of their current consumption values. Also, while C18 is the periodic version of $\mathrm{C} 16$, there is no significant variation in the battery time-to-failure. Even inserting a 50-minute rest period in case C17 does not yield adequate lifetime improvements over case C16. Finally, note that in $\mathrm{C} 19$ is derived from $\mathrm{C} 18$ by replacing WAV1 and Talk1 by WAV2 and Talk3, respectively. Although WAV2 and Talk 3 consume less power than WAV 1 and Talk1, the lifetimes in cases $\mathrm{C} 19$ and $\mathrm{C} 18$ are almost the same.

Our predictions for the Itsy battery produce the same lifetimes for cases $\mathrm{C} 15, \mathrm{C} 16$, and $\mathrm{C} 18$, as expected. The improvement in C19 over C18 is only $2 \%$ (216.4 minutes vs 211.4 minutes). In case $\mathrm{C} 17$, the 50-minute delay due to the inserted rest period directly translated into the 50-minute delay for the time-to-failure (no recovery predicted).

Summary: It is clear that the real Itsy battery and the simulated DUALFOIL battery behave differently. The former exhibits much weaker nonlinearity than the latter. As a result, the dependency of the Itsy battery behavior on a timing characteristics of a load profile (e.g. insertion of off-line periods) is not as strong as for the case of the simulated battery.

The DUALFOIL battery may or may not exist in reality. If such a battery exists, then our lifetime predictions are of significant practical value. Otherwise, our results should be treated as a demonstration of the high quality of our model with respect to variations in $\beta$ - the parameter quantifying battery nonlinearity.

\section{CONCLUSION}

We described an analytical model of a generic battery for use in portable system/application development. The proposed model was thoroughly evaluated with respect to the low-level numerical simulator DUALFOIL for lithium-ion cells. Thirty-two constant load tests and twenty-two variable load tests were conducted with the DUALFOIL. These tests were selected to reflect typical applications run on a pocket computer. For the constant loads, predictions were within $10 \%$ error margin, and for the variable loads, the maximum error was less than $5 \%$. For a subset of the test profiles, our model was also verified against a real battery used in the Itsy pocket computer.

\section{REFERENCES}

[1] P. Arora, M. Doyle, A. Gozdz, R. White, and J. Newman. Comparison between computer simulations and experimental data for high-rate discharges of plastic lithium-ion batteries. J. Power Sources, 88, 2000.

[2] A. Bard and L. Faulkner. Electrochemical Methods. Wiley, New York, 1980.

[3] L. Benini, G. Castelli, A. Macii, E. Macii, M. Poncino, and R. Scarsi. A discrete-time battery model for high-level power estimation. Proc. DATE, 2000.

[4] M. Doyle, T. Fuller, and J. Newman. Modeling of galvanostatic charge and discharge of the lithium/polymer/insertion cell. J. Electrochem. Soc., 140(6), 1993.

[5] M. Doyle and J. Newman. Modeling the performance of rechargeable lithium-based cells: Design correlations for limiting cases. J. Power Sources, 54, 1995.

[6] M. Doyle and J. Newman. Comparison of modeling predictions with experimental data from plastic lithium ion cells. J. Electrochem. Soc., 143(6), 1996.

[7] T. Fuller, M. Doyle, and J. Newman. Simulation and optimization of the dual lithium ion insertion cell. $J$. Electrochem. Soc., 141(1), 1994.

[8] S. Gold. A PSPICE macromodel for lithium-ion batteries. Proc. Battery Conf., 1997.

[9] D. Linden. Handbook of Batteries. McGraw-Hill, New York, 1995.

[10] T. Martin. Balancing Batteries, Power, and Performance: System Issues in CPU Speed-Setting for Mobile Computing. Dissertation, Carnegie Melon University, 1999.

[11] D. Panigrahi, C. Chiasserini, S. Dey, R. Rao, A. Raghunathan, and K. Lahiri. Battery life estimation of mobile embedded systems. Proc. VLSI Design, 2001.

[12] M. Pedram and Q. Wu. Design considerations for battery-powered electronics. Proc. DAC, 1999.

[13] D. Rakhmatov and S. Vrudhula. An analytical high-level battery model for use in energy management of portable electronic systems. Proc. ICCAD, 2001.

[14] T. Simunic, L. Benini, and G. D. Micheli. Energy-efficient design of battery-powered embedded systems. IEEE Trans. VLSI Systems, February 2001.

[15] M. Viredaz and D. A. Wallach. Power evaluation of a handheld computer: a case study. Compaq WRL Research Report 2001/1, May 2001.

[16] D. A. Wallach. Interpreting the battery lifetime of the Itsy version 2.4. Compaq WRL Technical Note TN-61, December 2001. 